\title{
Clustering and Pattern Formation in Chemorepulsive Active Colloids
}

\author{
Benno Liebchen, ${ }^{1, *}$ Davide Marenduzzo, ${ }^{1, \dagger}$ Ignacio Pagonabarraga, ${ }^{2, *}$ and Michael E. Cates ${ }^{3, \S}$ \\ ${ }^{1} S U P A$, School of Physics and Astronomy, University of Edinburgh, Edinburgh EH9 3FD, United Kingdom \\ ${ }^{2}$ Departament de Física Fonamental, Universitat de Barcelona-Carrer Martí i Franquès 1, 08028-Barcelona, Spain \\ ${ }^{3}$ DAMTP, Centre for Mathematical Sciences, University of Cambridge, Cambridge CB3 OWA, United Kingdom
}

(Received 13 October 2015; published 16 December 2015)

\begin{abstract}
We demonstrate that migration away from self-produced chemicals (chemorepulsion) generates a generic route to clustering and pattern formation among self-propelled colloids. The clustering instability can be caused either by anisotropic chemical production, or by a delayed orientational response to changes of the chemical environment. In each case, chemorepulsion creates clusters of a self-limiting area which grows linearly with self-propulsion speed. This agrees with recent observations of dynamic clusters in Janus colloids (albeit not yet known to be chemorepulsive). More generally, our results could inform design principles for the self-assembly of chemorepulsive synthetic swimmers and/or bacteria into nonequilibrium patterns.
\end{abstract}

PACS numbers: 82.70.Dd, 05.65.+b, 82.40.Ck, 87.17.Jj

Active systems, such as suspensions of autophoretic colloidal swimmers, motile bacteria, or other self-propelled particles, are far from equilibrium even in steady state due to their continuous energy expenditure [1,2]. Unlike isothermal Brownian colloids, motile particles can accumulate in regions where they move more slowly. They also slow down at high density, creating a positive feedback loop that can lead ultimately to motility-induced phase separation (MIPS) $[3,4]$.

Experiments with artificial self-propelled colloids have reported self-organized dynamic clustering, sometimes at densities well below that expected to trigger MIPS $[5,6]$. These "living clusters" seem to reach a limiting size and do not coarsen indefinitely: they show microphase separation rather than macrophase separation as in MIPS. So far, the underlying mechanism remains unclear.

In such experiments, motility is autophoretic: a chemical reaction is catalyzed on part of the colloid surface, creating a gradient of reagent and/or product that drives the particle forward by diffusiophoresis or a similar mechanism [7-9]. The same gradients can then cause chemically mediated long-range interactions between the colloids, and can also cause rotational torques that bias the swimming direction up or down the chemical gradient (an effect known as chemotaxis) [10,11]. This fact has suggested a parallel between the experiments in Ref. [5] and the Keller-Segel (KS) model [12-14] for microorganisms interacting via chemical signaling. This mapping, which assumed that active colloids swim up chemical gradients (the "chemoattractive" case), can explain clustering, but leads to complete phase separation, rather than a self-limiting cluster size. A combination of a passive drift up the chemical gradient and self-propulsion down it ("chemorepulsion") might lead to finite size clusters [15]; however, at variance with experiments $[5,16]$, these should shrink as the self-propulsion speed increases [15]. A more general study of chemoresponsive active colloids in the limit of fast chemical dynamics suggests a far larger potential for pattern formation than is predicted by the KS model [10].

Here we propose a theoretical framework for active colloids that describes at a continuum level not only colloidal and chemical densities, but also the local mean orientation ("polarization") of the active particles. We thereby uncover new instability mechanisms which show that chemorepulsion can lead to clustering and pattern formation. These mechanisms were largely ignored in the literature so far, perhaps because chemoattraction offers a more obvious route to (bulk) phase separation.

We call our two mechanisms the "Janus instability" and the "delay-induced instability." The physics underlying these is indicated in Figs. 1(b) and 1(c), respectively. For the Janus instability, active particles reorient and move towards a fluctuation-induced minimum in the chemical density, thereby forming an inward-pointing aster [Fig. 1(b)]. Because of local anisotropy of the chemical production (inevitable for Janus colloids half-coated with catalyst), this cluster produces a shell of chemorepellent, keeping the particles within it together and driving others away, so that it cannot grow beyond a certain size. In the delay-induced instability, which appears in a different parameter regime, colloids respond differently to the same fluctuation. Initially they accumulate at the chemical minimum, producing more chemical and lifting the concentration there [Fig. 1(c)]. If they reorient slowly on the time scales needed to cancel the initial fluctuation, the production can overshoot and the minimum becomes a maximum before particles finally drift away. This can trigger a cyclical instability towards traveling wave patterns. When both instability mechanisms act together, we 

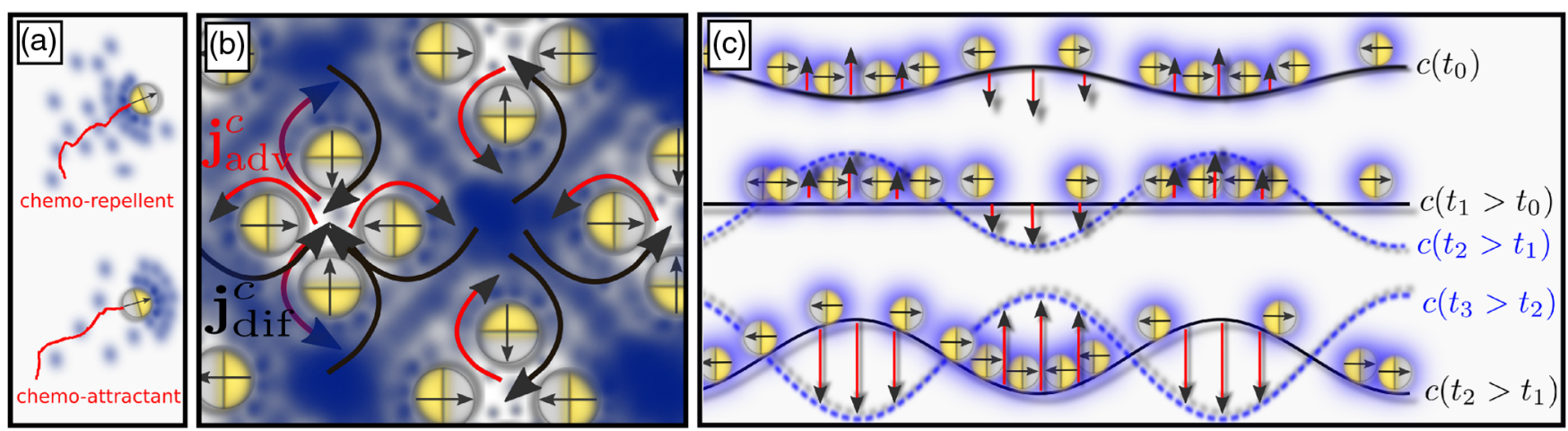

FIG. 1 (color online). Schematics: (a) Colloidal Janus particles (spheres) half-coated with a catalytic material (yellow) that produces a chemical species (blue); the colloids self-propel by autophoresis in the resulting gradient. Chemorepellent colloids propel from high to low concentration of the chemical, chemoattractant colloids vice-versa. (b) Anisotropy-induced Janus instability and clusters of selflimiting size. (c) Delay-induced instability, which can lead to traveling wave patterns.

predict "blinking" patterns where clusters continually exchange particles and never reach a steady state.

These chemorepulsive instabilities may shed light on the dynamic clustering or microphase separation recently observed in experiments $[5,6,16]$. Both instability mechanisms induce clusters of a self-limiting area that grows with self-propulsion speed, which qualitatively agrees with experiments $[5,16]$. More generally, chemorepulsive instabilities suggest new design principles for the active selfassembly of colloids into spatiotemporal patterns with tunable properties. Our key message that chemorepulsion can generate instabilities of uniform states could also be relevant for biophysics, where the chemoattractive KS instability has long been assumed to drive structure formation among microorganisms [17-19].

We describe active colloids (living or artificial) at a coarse-grained level, through their density and polarization fields, $\rho(\mathbf{x}, t)$ and $\mathbf{p}(\mathbf{x}, t)$. The latter is a local average of the unit vector describing the propulsive direction; this rotates in response to gradients of a chemical density field $c(\mathbf{x}, t)$. The colloids self-propel at constant speed $v_{0}$ and also have isotropic diffusivity $D_{\rho}$. To represent autophoretic colloids (or signaling bacteria), we assume that the chemical species is produced by the colloids at local rate $k_{0} \rho$ and decays at rate $k_{d}$, leading to

$$
\begin{aligned}
& \dot{\rho}=-\nabla \cdot\left(\rho v_{0} \mathbf{p}\right)+D_{\rho} \nabla^{2} \rho, \\
& \dot{\mathbf{p}}=-\gamma \mathbf{p}+D_{p} \nabla^{2} \mathbf{p}+\beta \nabla c-\gamma_{2}\left|\mathbf{p}^{2}\right| \mathbf{p}, \\
& \dot{c}=D_{c} \nabla^{2} c+k_{0} \rho-k_{d} c+k_{a} \nabla \cdot(\rho \mathbf{p}) .
\end{aligned}
$$

Here $\beta$ measures the chemotactic coupling strength; when positive, this represents chemoattraction [Fig. 1(a)], for instance bacteria swimming up food or aspartate gradients $[18,20]$. Here, however, we focus on negative $\beta$, describing chemorepulsion, as arises for at least some types of colloid with nonuniform surface mobility [10], or for cells fleeing from toxins [18]. In Eq. (1) the polarization decays locally at a relaxation rate $\gamma$, set by rotational diffusion; it also has translational diffusivity $D_{p} \sim D_{\rho} \sim v_{0}^{2} / \tau$ [4], which smears out details of $\mathbf{p}$ on scales below the "run length" $v_{0} / \gamma$. The term in $\gamma_{2}$ describes saturation in $\mathbf{p}$ at strong alignment. Finally, $k_{a} \nabla \cdot(\rho \mathbf{p})$, where $k_{a}$ has the dimensions of speed, describes an anisotropic correction to the isotropic chemical production term $\left(k_{0} \rho\right)$, arising whenever the chemical is produced by the colloid asymmetrically [21].

Rewriting Eq. (1) for dimensionless quantities $\tilde{t}=k_{d} t$, $\tilde{x}=x \sqrt{k_{d} / D_{\rho}}$, and setting $\tilde{\rho}=\rho k_{0}|\beta| v_{0} /\left(k_{d}^{2} D_{\rho}\right), \tilde{\mathbf{p}}=$ $v_{0} /\left(\sqrt{k_{d} D_{\rho}}\right) \mathbf{p}, \quad \tilde{c}=c v_{0}|\beta| /\left(k_{d} D_{\rho}\right)$ we show (in the Supplemental Material [22]) that the parameter space is spanned by five dimensionless variables $\Gamma=\gamma / k_{d}$, $\mathcal{D}_{p}=D_{p} / D_{\rho}, \quad \mathcal{D}_{c}=D_{c} / D_{\rho}, \quad \kappa=k_{a} k_{d} /\left(k_{0} v_{0}\right), \quad$ and $\Gamma_{2}=\gamma_{2} D_{\rho} / v_{0}^{2}$. Also $s=\operatorname{sgn}(\beta)$ distinguishing positive $(s=1)$ and negative $(s=-1)$ chemotaxis. In the following, we omit tildes.

We have solved Eq. (1) numerically on a square box of side $L$ by finite difference methods using periodic boundary conditions and a small perturbation of the uniform state $(\rho, \mathbf{p}, c)=\left(\rho_{0}, \mathbf{0}, \rho_{0}\right)$ as initial conditions.

With chemoattraction $(s=1)$, the initial uniform state is stable for small $\rho_{0}$, whereas for stronger coupling it is unstable to the formation of dense colloidal clusters that colocalize with maxima in $c$ [Figs. 2(a) and 2(b)]. These droplets coarsen continuously to yield complete phase separation at late times, albeit featuring orientational order in the form of a macro-aster [Fig. 2(d), inset]. This chemically induced phase separation is well understood [14]: colloids swim towards high chemical concentration forming a cluster, which increases chemical production locally, recruiting further particles, etc.

Although this feedback loop is absent for chemorepulsion $(s=-1)$, upon tuning $\rho_{0}$ beyond a certain threshold, we observe, strikingly, that the initial fluctuations amplify also for chemorepulsive colloids. In marked contrast with the chemoattractive case, the resulting dense colloidal clusters do not coarsen beyond a characteristic size 

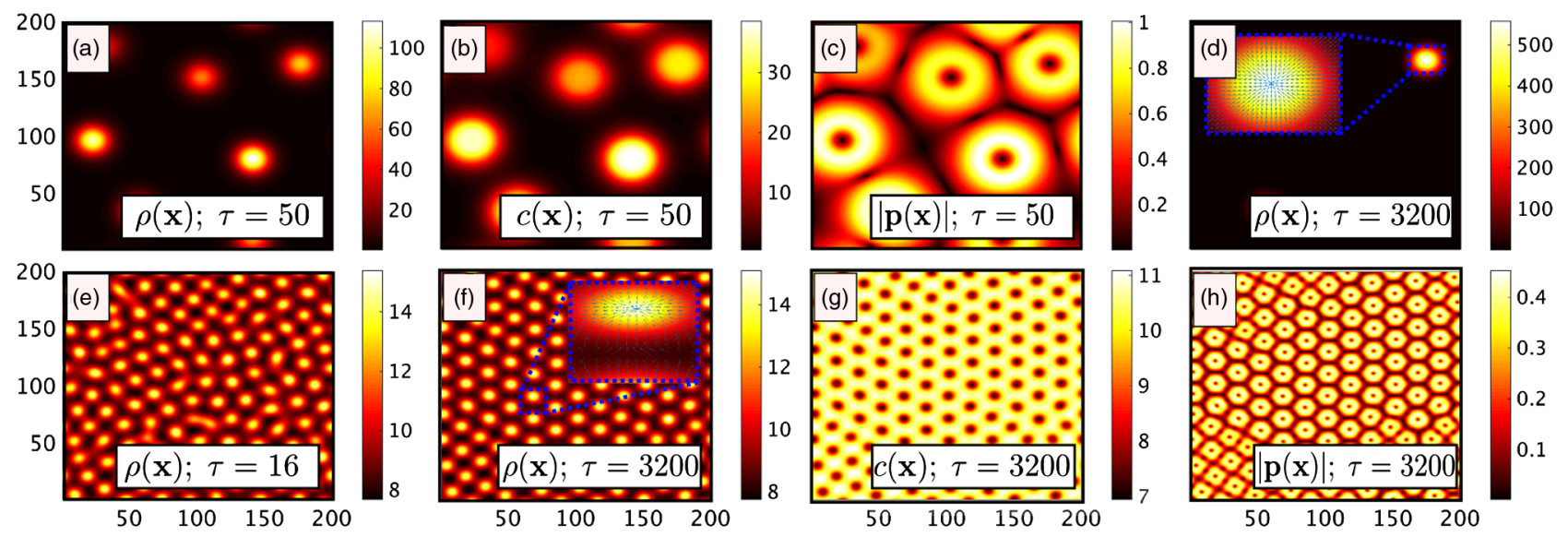

FIG. 2 (color online). Time evolution of the density fields $\rho, c,|\mathbf{p}|$ (insets: $\mathbf{p} /|\mathbf{p}|$ ) for a weakly perturbed uniform initial state (color shows value of the fields). (a)-(d): Clustering and phase separation for chemoattractive colloids $\left(s=1, \rho_{0}=8\right)$. (e)-(h): Arrested phase separation and stationary density pattern for chemorepulsive colloids resulting from the Janus instability $\left(s=-1, \rho_{0}=10\right)$. Other parameters: $\mathcal{D}_{p}=\mathcal{D}_{c}=\kappa=\Gamma=1, \Gamma_{2}=10$ and $x_{u}=5 ; t_{u}=1$. (Time and space units of $\tau=t / t_{u}$ and $x=x / x_{u}$ ).

[Figs. 2(e)-2(h) and Supplemental Material [22], Videos $1,4,5]$. The behavior en route to this steady state is complex; it can feature amplitude oscillations, or "blinking” (see Supplemental Material [22], Videos 1,4,5). Blinking clusters dynamically exchange particles, before settling down into a stationary arrangement, which normally consists of a hexagonal lattice of droplets and an inverted pattern in the chemical density [Fig. 2(g)]. Deviations from the ideal hexagonal structure [Fig. 2(h)] can be more or less pronounced, depending on the specific parameter choices. As can be seen in Fig. 2(f) (inset), chemorepulsive colloids point towards the chemical density minima between the colloidal clusters, and the overall orientational pattern consists of stable asters and anti-asters. Parameter fine tuning, or choosing no-flux boundary conditions instead of periodic ones, can lead instead to seemingly irregular cluster distributions or permanent blinking (Supplemental Material [22], Videos 4 and 5).

Intriguingly, we also find colloidal waves and traveling oscillatory patterns. In particular, upon further increasing the overall density $\rho_{0}$ we observe an amorphous pattern which evolves towards a more regular state, where clusters continuously merge, split and decay (Fig. 3 and Supplemental Material [22], Videos 2 and 3). Eventually, the colloidal and chemical density fields may approach rectangular latticelike patterns, which phase-lock and travel at constant velocity along a common direction. The selection of a simple traveling wave is favored by periodic boundary conditions and small system sizes (Supplemental Material [22], Video 2); when choosing large systems (Supplemental Material [22], Video 3), or imposing no-flux boundary conditions (which might better represent experiments) the pattern persists as a flowing non-periodic state, continuously forming clusters of well-defined size and amplitude (Supplemental Material [22], Video 4).

To further understand the mechanisms of chemorepulsive pattern formation, we have performed a linear stability analysis of our model. Assuming that $\mathbf{p}$ relaxes fast compared to $\rho$ and $c$ and $\Gamma_{2} \ll 1$ and $\mathcal{D}_{p} \ll 1$ we find $\mathbf{p}=(s / \Gamma) \nabla c$ (see Supplemental Material [22]) and obtain, after rescaling variables $\rho^{\prime} \equiv \rho / \Gamma, c^{\prime} \equiv c / \Gamma$, a generalized KS (GKS) model $[12,14,20]$ that accounts both for chemorepulsion $(s=-1)$, and anisotropic chemical production $(\kappa \neq 0)$ :

$$
\begin{aligned}
& \dot{\rho}=-s \nabla \cdot(\rho \nabla c)+\nabla^{2} \rho, \\
& \dot{c}=s \kappa \nabla \cdot(\rho \nabla c)+\mathcal{D}_{c} \nabla^{2} c+\rho-c .
\end{aligned}
$$
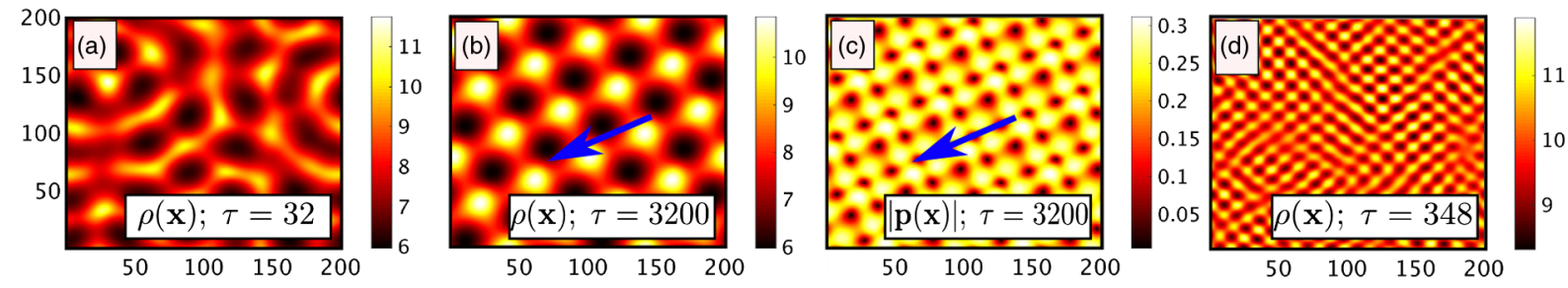

FIG. 3 (color online). As Fig. 2, for $s=-1, \rho_{0}=8$. The snapshots show the amorphous transient dynamics (a), traveling wave patterns (b),(c) and continuously moving states (d) for chemorepulsive colloids resulting from the delay-induced instability. Other parameters as in Fig. 2 for (a)-(c) and $\mathcal{D}_{c}=0.2 ; \mathcal{D}_{p}=1 ; \kappa=0.5 ; \Gamma=1.0 ; \Gamma_{2}=6.0$ and $x_{u} \approx 1.83 ; t_{u}=1$ for $(\mathrm{d})$. 
For $s=1$, linear stability analysis (see Supplemental Material [22]) reveals the standard chemoattractive KS instability when $\rho_{0}>1$ (or in physical units, when $\left.\rho_{0}>\left(D_{\rho} k_{d} \gamma / k_{0} \beta v_{0}\right)\right) \quad[14,20]$. For chemorepulsion $(s=-1)$ we find a qualitatively different picture, in which instability arises when $\kappa \rho_{0}>\mathcal{D}_{c}$, or, in physical units, when $k_{a} \beta \rho_{0}>\gamma D_{c}$. In contrast to the attractive case, the instability is now determined by the anisotropic reaction term; it disappears for isotropic active colloids, $k_{a} \rightarrow 0$. Hence we refer to this as the "Janus instability." In marked contrast to the classical KS case for chemoattraction, this is a short wavelength instability, arising only for $q>q^{*}=$ $\sqrt{\left(1+\rho_{0}\right) /\left(\kappa \rho_{0}-\mathcal{D}_{c}\right)}$ (Fig. 1 in the Supplemental Material [22]). For large $\rho_{0}$, or large $\kappa$, the corresponding length scale is $l^{*} \sim \kappa^{1 / 2}\left[l^{*} \sim \sqrt{\left(D_{\rho} k_{a}\right) /\left(k_{0} v_{0}\right)}\right.$ in physical units], and diverges when the isotropic production rate vanishes. Remarkably, the steady-state cluster domain area grows as $l^{* 2} \propto v_{0}$ (assuming $D_{\rho} \propto v_{0}^{2}[4,5]$ ), a prediction which turns out to remain valid for Eqs. (1) even with finite values of $D_{p} \propto v_{0}^{2}$. This may explain recent observations of self-limiting active clusters, whose particle number increases linearly with $v_{0}[5,16]$.

Why does the Janus instability lead to arrested, not full, phase separation? We find that two effects limit cluster growth. First, each cluster creates a shell of chemorepellent which drives away colloids passing nearby, hindering their arrival. Second, as the cluster increases in size, so does the quantity of chemorepellent created at its core via the isotropic production term: once too large, this disintegrates the cluster. Note that the GKS model predicts a short wavelength divergence of the growth rate, $\operatorname{Re}[\lambda(\mathbf{q})]$ as $|\mathbf{q}| \rightarrow \infty$ which is generally prevented by rotational dynamics as we show in the Supplemental Material [22].

A general, numerical evaluation of the dispersion relations $\lambda(q)_{1,2,3}$ for the three distinct modes that emerge from the full model Eq. (1) allows us to plot phase diagrams on the $\rho_{0}, \kappa$ plane; a typical example is shown in Fig. 4. In general, such phase diagrams show three different regimes: (i) one in which the uniform state is stable (black in Fig. 4); (ii) one in which the growth rate of the instability is real and positive, which corresponds to the Janus instability (orange to white); and (iii) one in which there is a nonzero imaginary part in the growth rate (purple with green stars). Modulo small corrections from finite-size effects, the length scales set by the wave vectors of maximal growth rate closely match those observed in the simulations of Figs. 2 and 3.

Remarkably, the phase diagram shows that the oscillatory instability can develop even for isotropic chemical production, $\kappa=0$, at large colloidal densities (red ellipse in Fig. 4). Accordingly we must have a second instability, distinct from the Janus mechanism which requires finite $\kappa$. (The cyan line in Fig. 4 does not reach $\kappa=0$ at finite $\rho_{0}$.) This can be traced to the finite relaxation rate of $\mathbf{p}$ which

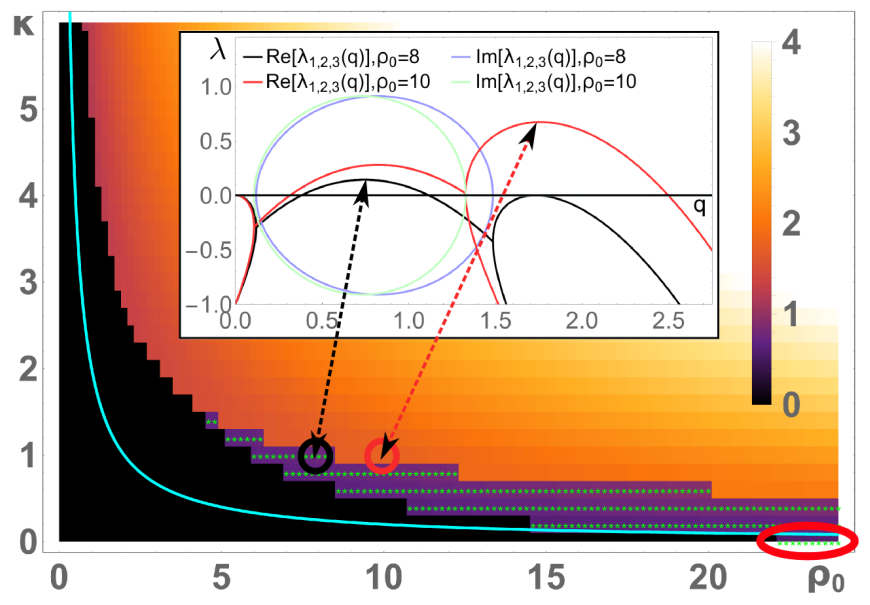

FIG. 4 (color online). Phase diagram showing instabilities of the uniform state for Eqs. (2) and $\mathcal{D}_{c}=\mathcal{D}_{p}=\Gamma=1$. Black denotes stability; colors show the wavelength of the fastest growing unstable mode and green stars show where this mode is oscillatory. The red ellipse highlights instability for $\kappa=0$. The cyan line shows the onset of instability as predicted by the GKS model Eq. (2). Circles mark the parameter sets of Figs. 2(e)-2(h) and Figs. 3(a)-3(c). Inset: Dispersion relation for the encircled parameters (a third solutions, not shown, is real and negative). Upper arrow heads: fastest growing mode.

we neglected when deriving the GKS model Eq. (2). Because the oscillatory unstable mode requires in effect a delayed reorientation of $\mathbf{p}$, we name this the "delayinduced instability." Intuitively, it can be understood as follows [see the simplified, 1D cartoon in Fig. 1(c)]. Chemorepulsive colloids move towards the minima of an initial fluctuation in chemical density (upper panel, solid line), accumulate there, and produce chemicals opposing the original fluctuation. Because of a finite response time this production does not stop at uniform density (middle panel) but overshoots, leading to reversal and possible amplification of the initial fluctuation (dashed blue line). This cycle repeats (lower panel) and represents a delayinduced feedback loop: an initial fluctuation of the chemical density field triggers another fluctuation of the same field but with opposite sign.

To understand the delay-induced instability quantitatively, we develop a minimal model in the Supplemental Material [22], showing that consecutive fluctuations can amplify if $\rho_{0}>1$, leading to an oscillatory instability. Deep in the pattern forming regime $\left(\rho_{0} \gg 1\right)$ we find that the wavelength of the fastest growing mode scales as $\rho_{0}^{1 / 2}$, or in physical units as $l^{*} \propto \sqrt{|\beta| v_{0} k_{0} \rho_{0} / k_{d}^{3}}$ predicting (in accordance with our numerical simulations) that the cluster area grows linearly with the self-propulsion velocity.

The transient or permanent "blinking" of clusters discussed above appears close to the transition line between the Janus instability and the delay-induced instability (Fig. 4). Here, stationary and oscillatory modes of different 
wavelength grow at a similar rate out of the uniform state and lead to an effective particle motion on top of a stationary density profile which causes the blinking. (See Supplemental Material [22]).

In conclusion, our two chemorepulsive instabilities create a robust new route to pattern formation. Both instabilities lead to clusters of self-limiting area which grows linearly with propulsion speed $v_{0}$. This agrees with recent experimental observations $[5,6,16]$ and may shed light on the still mysterious mechanism underlying their appearance. (Competing explanations based on the chemoattractive KS instability either predict macrophase separation [26] or clusters shrinking with increasing $v_{0}$ [15].) Although we mainly report regular patterns in our figures, our model suggests that irregular cluster arrangements, more akin to patterns found in experiments with active colloids, can also be found depending on location in parameter space and boundary conditions (Videos 3-5, Supplemental Material [22]). More generally, our chemorepulsive instabilities might inform design principles for creating active colloids that can self-assemble into spatiotemporal patterns with desired properties. Finally, our key finding that chemorepulsion can generate the instability of uniform states might also be important for biophysics, where the chemoattractive KS instability has long been invoked to explain patterns of microorganisms [17-19]. In growing biofilms, for example, the interaction of bacteria with a self-secreted polymer [27] might be interpreted as chemorepulsion.

We thank EPSRC EP/J007404 for funding. B. L. gratefully acknowledges funding by a Marie Skłodowska-Curie Intra European Fellowship (G. A. No. 654908) within Horizon 2020. M. E. C. is funded by a Royal Society Research Professorship. I. P. acknowledges the Dirección General de Investigación (Spain) and DURSI for financial support under Projects No. FIS 2011-22603 and No. 2014SGR-922, respectively, and Generalitat de Catalunya under program Icrea Acadèmia.

*Benno.Liebchen@staffmail.ed.ac.uk

†marendu@ph.ed.ac.uk

*ipagonabarraga@ub.edu

\$m.e.cates@damtp.cam.ac.uk

[1] M. C. Marchetti, J. F. Joanny, S. Ramaswamy, T. B. Liverpool, J. Prost, M. Rao, and R. A. Simha, Rev. Mod. Phys. 85, 1143 (2013).
[2] S. Ramaswamy, Annu. Rev. Condens. Matter Phys. 1, 323 (2010).

[3] J. Tailleur and M. Cates, Phys. Rev. Lett. 100, 218103 (2008).

[4] M. E. Cates and J. Tailleur, Annu. Rev. Condens. Matter Phys. 6, 219 (2015)

[5] I. Theurkauff, C. Cottin-Bizonne, J. Palacci, C. Ybert, and L. Bocquet, Phys. Rev. Lett. 108, 268303 (2012).

[6] J. Palacci, S. Sacanna, A. P. Steinberg, D. J. Pine, and P. M. Chaikin, Science 339, 936 (2013).

[7] R. Golestanian, T. B. Liverpool, and A. Ajdari, Phys. Rev. Lett. 94, 220801 (2005).

[8] R. Golestanian, T. B. Liverpool, and A. Ajdari, New J. Phys. 9, 126 (2007).

[9] A. Brown and W. Poon, Soft Matter 10, 4016 (2014).

[10] S. Saha, R. Golestanian, and S. Ramaswamy, Phys. Rev. E 89, 062316 (2014).

[11] Y. Hong, N. M. K. Blackman, N. D. Kopp, A. Sen, and D. Velegol, Phys. Rev. Lett. 99, 178103 (2007).

[12] E. F. Keller and L. A. J. Segel, Theor. Popul. Biol. 26, 399 (1970).

[13] E. F. Keller and L. A. J. Segel, Theor. Popul. Biol. 30, 225 (1971).

[14] M. Meyer, L. Schimansky-Geier, and P. Romanczuk, Phys. Rev. E 89, 022711 (2014).

[15] O. Pohl and H. Stark, Phys. Rev. Lett. 112, 238303 (2014).

[16] I. Buttinoni, J. Bialké, F. Kümmel, H. Löwen, C. Bechinger, and T. Speck, Phys. Rev. Lett. 110, 238301 (2013).

[17] G. Gerisch, Annu. Rev. Physiol. 44, 535 (1982).

[18] H. C. Berg, E. coli in Motion. (Springer, New York, 2004).

[19] M. J. Tindall, P. K. Maini, S. L. Porter, and J. P. Armitage, Bull. Math. Biol. 70, 1570 (2008).

[20] J. Murray, Mathematical Biology. II: Spatial Models and Biomedical Applications, 3rd ed. (Springer-Verlag, Berlin, 2003).

[21] Assuming a point source $-R \mathbf{p}$ away from the colloidal center, with $R$ as the colloid radius, the anisotropic term follows as lowest order correction (dipole) to the isotropic (monopole) field, with $k_{a} \sim k_{0} R$.

[22] See Supplemental Material at http://link.aps.org/ supplemental/10.1103/PhysRevLett.115.258301 for details on the linear stability analysis and the amplitude blinking mechanism, which includes Refs. [23-25].

[23] A. Hurwitz, Math. Ann. 46, 273 (1895).

[24] J. L. Willems, Stability Theory of Dynamical Systems (Wiley Interscience Division, New York, 1970).

[25] A. M. Turing, Phil. Trans. R. Soc. A, 237, 37 (1952).

[26] This would require kinetic hindrance of cluster growth to explain experiments.

[27] P. Ghosh, J. Mondal, E. Ben-Jacob, and H. Levine, Proc. Natl. Acad. Sci. U.S.A. 112, E2166 (2015). 